\title{
Experimental Pathology, Tumors
}

National Cancer Institute

\section{Source}

National Cancer Institute. Experimental Pathology, Tumors. NCI Thesaurus. Code C18782.

The study of the neoplastic process by studying artificially produced tumors. 